\title{
CONSTRUCTION OF VOLATILITY INDICES USING A MULTINOMIAL TREE APPROXIMATION METHOD
}

\author{
Dragos Bozdog, Ionuţ Florescu, \\ Khaldoun Khashanah, and Hongwei Qiu \\ Dept. of Mathematical Sciences, Stevens Institute of Technology, \\ Hoboken, NJ 07030, USA
}

ver. 15:57, Wednesday $16^{\text {th }}$ February, 2011

\begin{abstract}
This paper introduces a new methodology for an alternative calculation of market volatility index based on a multinomial tree approximation of a stochastic volatility model. The estimation is performed by constructing synthetic options with consistent properties. Several variants of this index are calculated and their performance is analyzed over the whole dataset and over a subset of data corresponding to particular market events. The proposed index is compared with the VIX produced by CBOE.
\end{abstract}

\section{Introduction}

The Chicago Board Options Exchange (CBOE) Market Volatility Index (VIX) was introduced by professor Robert Whaley in 1993. It is a forward-looking index that provides the expected stock market volatility over the next 30 calender days. There were two purposes for creating this index. First, it was intended to provide a benchmark of the expected short-term market volatility. And second, it was created to provide an index on which options and futures contracts can be written. In 2003 the methodology was revised and VIX started trading future contracts in 2004. 
The VIX index is computed directly from options written on the Standards and Poor 500 equity index (SPX) or the ETF that tracks it (the SPY), thus its movement is determined by the market demand of calls and puts written on the S\&P500. Indeed, Bollen and Whaley (2004) show that the demand to buy out-of-the-money and at-the-money SPX puts is a key driver in the movement in SPX implied volatility measures such as VIX. The reason given is that the option market became dominated by portfolio insurers or hedgers, who routinely buy out-of-the-money and at-the-money SPX index put options for insurance purposes to protect their portfolio from a potential market crash. Very often, the VIX index was termed as an "Investor Fear Gauge". This is due to the fact that the VIX spikes during periods of market turmoil.

\subsection{Calculation of VIX by CBOE}

The current calculation of VIX by CBOE is based on the concept of fair value of future variance developed by Demeterfi et al. (1999). The fair value of future variance is determined from market observables such as option prices and interest rates independent of any option pricing model. The fair value of future variance is defined as following (equation 26 in Demeterfi et al. (1999)):

$$
\begin{gathered}
K_{v a r}=\frac{2}{T}\left\{r T-\left[\frac{S_{0}}{S_{*}} e^{r T}-1\right]-\log \left(\frac{S_{*}}{S_{0}}\right)+e^{r T} \int_{0}^{S_{*}} \frac{P(T, K)}{K^{2}} d K\right. \\
\left.+e^{r T} \int_{S_{*}}^{\infty} \frac{C(T, K)}{K^{2}} d K\right\}
\end{gathered}
$$

where $S_{0}$ is the current asset price, $C$ and $P$ are call and put prices, respectively, $r$ is the risk-free rate, $T$ and $K$ are option maturity and strike price, respectively, and $S_{*}$ is an arbitrary stock price which is typically chosen to be close to the forward price.

The calculation of VIX by CBOE follows equation (1), as explained in the CBOE white paper (see CBOE (2003)). CBOE calculates the VIX index using the following formula:

$$
\sigma_{v i x}^{2}=\frac{2}{T} \sum_{i} \frac{\Delta K_{i}}{K_{i}^{2}} e^{r T} Q\left(T, K_{i}\right)-\frac{1}{T}\left(\frac{F_{0}}{K_{0}}-1\right)^{2}
$$


where

- $\sigma_{v i x}$ is the $\frac{V I X}{100}$.

- $T$ is the time to expiration.

- $F_{0}$ is the forward index level derived from index option prices.

- $K_{i}$ is the $i^{\text {th }}$ out-of-money option; a call if $K_{i}>F_{0}$ and a put if $K_{i}<F_{0}$.

- $\Delta K_{i}$ is the inverval between strike prices-half the distance between the strike on either side of,$K_{i}$ :

$$
\Delta K_{i}=\frac{K_{i+1}-K_{i-1}}{2}
$$

Please note that $\Delta K$ for the lowest or highest strike is simply the difference between the the lowest or highest strike and the next strike price.

- $K_{0}$ is the first strike below the forward index level.

- $r$ is the risk-free interest rate to expiration.

- And $Q\left(T, K_{i}\right)$ is the mid point of the bid-ask spread of each option with strike $K_{i}$.

A step by step calculation and an example that replicates the value of the VIX for a particular day is provided in the Appendix A.

\subsection{Issues with CBOE Procedure for VIX Calculation}

As shown by Jiang and Tian (2007), there are differences between the fair

value of future variance and the practical implementation of VIX provided by CBOE. The main differences come from the following:

(1) The calculation of fair value of future variance uses continuous strike prices. However, strike price vectors on CBOE or any other exchange are generally discrete.

(2) The calculation of fair value of future variance uses strike prices between 0 and $\infty$. But in reality, the strike prices have a small range. 
(3) The fair value of future variance produce the expected variance (volatility) corresponding to the maturity date of the options used. In reality, options with exact 30-day maturity are not available. VIX is obtained by linearly interpolating the variances of options with near term and next term maturity.

Due to the truncation of the data and the discreteness of the data, the CBOE procedure can generate errors (Jiang and Tian, 2007).

\section{New Methodology}

\subsection{Theoretical frame of stochastic volatility quadri- nomial tree method}

Stochastic volatility models have been proposed to better model the complexity of the market. The quadrinomial tree approximation developed by Florescu and Viens (2008) has demonstrated that it can be used to estimate option values and that it produces option chain values which are within bid ask spread. The quadrinomial tree method outperforms other approximating techniques (with the exception of analytical solutions) in terms of both error and time. In this work we use this methodology to approximate an underlying asset price process following:

$$
d X_{t}=\left(r-\frac{\varphi_{t}^{2}}{2}\right) d t+\varphi_{t} S_{t} d W_{t}
$$

where $X_{t}=\log S_{t}$ and $S_{t}$ is the asset price, $r$ is the short-term risk-free rate of interest, $W_{t}$ a standard Brownian Motions. $\varphi_{t}$ models the stochastic volatility process. It has been proved that for any proxy of the current stochastic volatility distribution at $t$ the option prices calculated at time $t$ converge to the true option prices (Theorem 4.6 in Florescu and Viens (2008)).

When using Stochastic volatility models we are faced with a real problem when trying to come up with ONE number describing the volatility. The model intrinsically has an entire distribution describing the volatility and therefore providing a number is nonsensical. However, since a number we need to provide the idea of this approach is to produce the price of a synthetic one month option with strike exactly the spot price and calculate the implied volatility value corresponding to the price we produce. The real challenge 
is to come up with a stochastic volatility distribution characteristic to the current market conditions.

In the current work we take the simplest approach possible. We use a proxy for this $\varphi_{t}$ calculated directly from the implied volatility values characterizing the option chains. This is used in conjunction with a highly recombining quadrinomial tree method to compute the price of options. The quadrinomial tree method is described in details in Florescu and Viens (2008). In the Appendix B we describe a one-step quadrinomial tree construction.

\subsection{Difference between CBOE Procedure and Quadri- nomial Tree Method}

(1) CBOE procedure calculates two variances from near term and next term out-of-money options chains and then it obtains the expected variance in 30-day by linearly interpolating the variance from near term and next term options chain. This arbitrary linear interpolation lacks a theoretical base. Using the quadrinomial tree method, we can compute the expected market volatility in exactly 30 days.

(2) Criteria for selection of the options used for the calculation are different. In CBOE procedure, the out-of-money options are first arranged according to the increase in strike price. All the individual options in the near term and next term out-of-money options are selected as long as two options next to each other don't have zero price at the same time. However, in our analysis we observe that some of the options selected are not actively traded and therefore their price may not reflect the expectation of the current market conditions. In the quadrinomial tree method, we select all the options (regardless of being out-of-money or not) as long as they reflect the market volatility. This is ensured by selecting all options with volume higher than 0 .

(3) In the CBOE procedure, 8 days before the maturity of the near term options chain, CBOE will roll over to the next/third options chains. The argument is that when the options are close to maturity, their price is more volatile and thus cannot represent the market volatility. However, it is also well known that options with larger maturities tends to have volatility close to the long term market mean volatility and this can lead to underestimating the market volatility. In the quadrinomial 
tree method, we use all the data of near term and next term options chains.

(4) In this work, we use all relevant options weighted accordingly. In theory all options with different maturities may be incorporated as they reflect the market volatility. Only the near term and next term options chains are used in this work, as from empirical studies including options with longer maturities have associated little weight and they do not generally influence the final result.

\section{Results and Discussions}

\subsection{Constructing a volatility index using different in- puts}

Options written on S\&P500 have three near term expiration months followed by three additional expiration months from the March quarterly cycle. We note that for the VIX calculation by CBOE, the closest near term options chain and the next term options chain are used. Also, when the maturity of the near term options is less than 8 days, next term options and the ones after will be used in the calculation. For the quadrinomial tree model, we explore the effects of using different option types and maturity on the resulting volatility index. In the next figures we compare constructed indices using five different ways.

(1) First, we consider only call options with the near term maturity and we obtain an index we denote cVIX-1. Figure 1(a) presents this index versus the CBOE VIX. It is evident that when only the near term options are used, the cVIX-1 is very unstable. When options are very close to maturity, they become more volatile and overestimate the true market volatility. This is the reason why CBOE avoids using options with maturity less than 8 days.

(2) Next we calculate cVIX-2, which uses only the next term call options chain (the maturity is larger than one month but less than two months). cVIX-2 is represented by the blue line in Figure 1(b). It appears that cVIX-2 has a similar pattern as that of the CBOE VIX. However, the value of cVIX-2 is generally smaller than that of VIX index. As we 

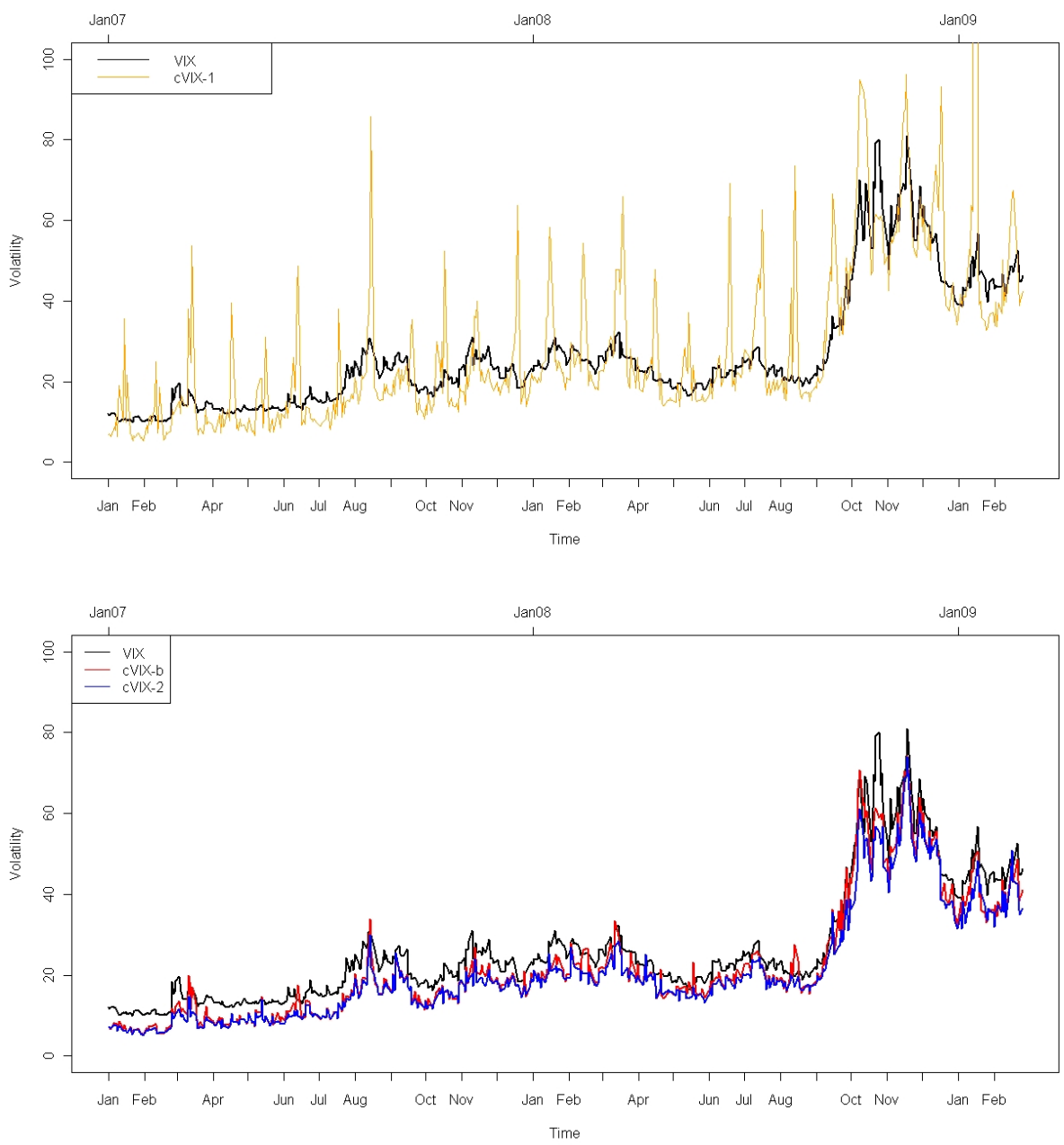

Figure 1: Comparison of VIX constructed using different methods: (a) cVIX1 which is computed from actively traded call options with the nearest maturity; (b) cVIX-2 (computed from actively traded call options with more than one-month but less than two-month maturity) and cVIX-b (constructed using call options with less than two-month maturity). 
shall see this is due to the fact that we only use data from call options in this calculation.

(3) In the third method, we use data from both call options chains (call options with the near term and next term maturities). However, the importance of data from these two options chains is different. The significance of each options chain is evaluated by a linear relationship depending on which maturity is closer to day 30th. This is ensured by setting the probability of using the near term option data as:

$$
p_{\text {near term }}=\left(30-T_{1}\right) /\left(T_{2}-T_{1}\right)
$$

where $T_{1}$ is the maturity of the near term options in days, and $T_{2}$ is the maturity of next term options in days. The probability of the next term values is $p_{\text {next term }}=1-p_{\text {near term }}$.

The index obtained by this method is denoted with cVIX-b and is shown in Figure 1(b) by the red line. We note that the cVIX-b has a similar pattern as the CBOE VIX. Similarly with the cVIX-2, the values are generally lower than the VIX. When comparing cVIX-b to cVIX2 , due to the effect of incorporation more volatile data, occasionally cVIX-b has higher value than cVIX-2, especially when there is a spike in the VIX value.

(4) As previously observed using only call options (cVIX-b or cVIX-2) tends to produce smaller values than the VIX. Thus, we next use only data coming from the put options. The computed pVIX-b uses both near term and next option with linear weight ratio, and is plotted in Figure 2. The figure clearly shows that the CBOE VIX values tend to be between the cVIX-b and the pVIX-b.

(5) Since the third and fourth methods produce indices that are bounding the CBOE VIX, we next are using both call options and put options. During the construction, since the number of call and put options are different, we set the probability of using the call data or put data proportional to their numbers. The probability of using near term options is determined by the same equations (5). The result, which is named as VIX', is shown in Figure 3(a) along with VIX. We also plot the arithmetic average of cVIX-b and pVIX-b (cpVIX). For clarity in Figure 3(b) we plot the differences between these indices. With the exception of the crash period the differences are consistent. 

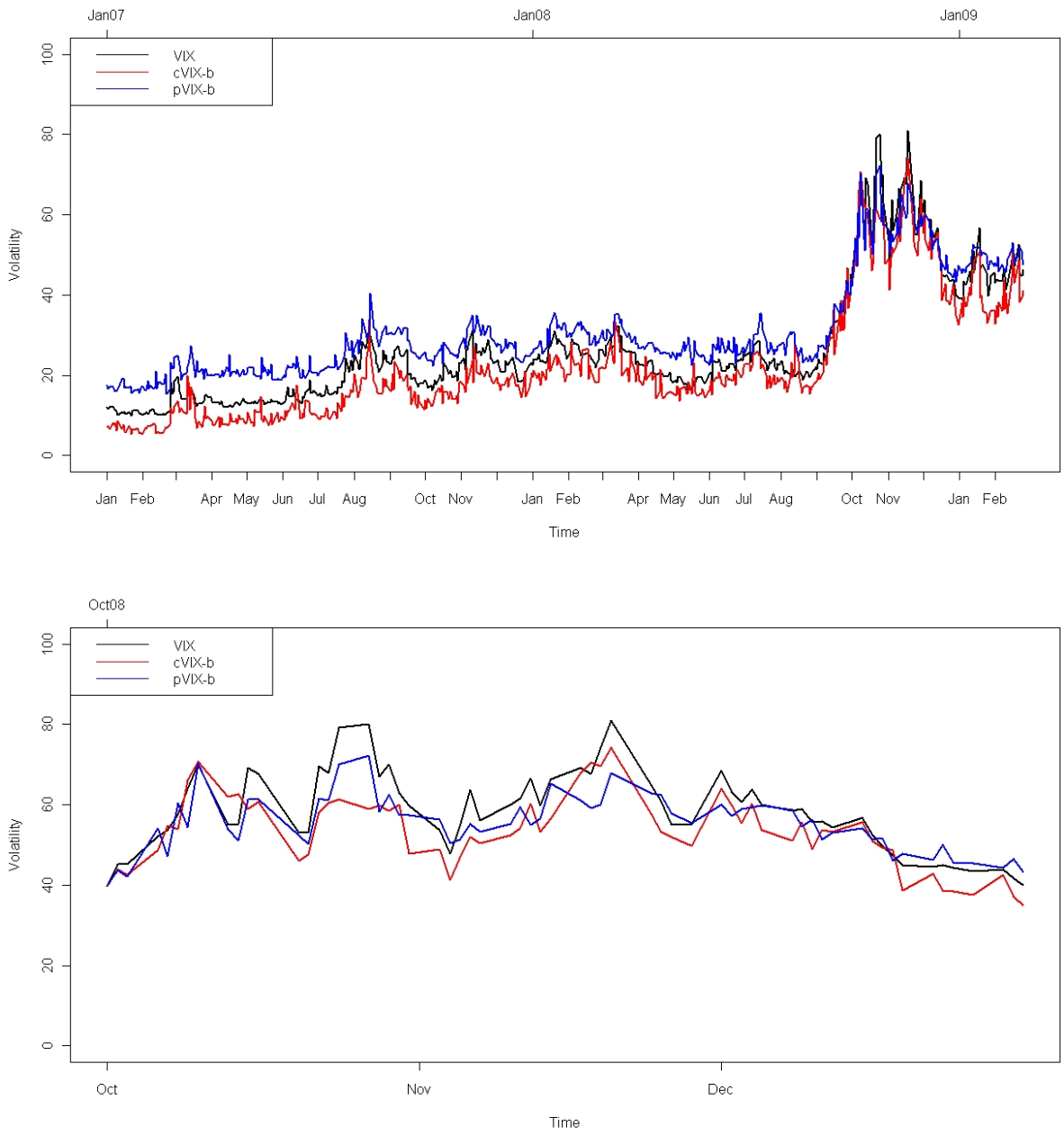

Figure 2: Comparison between constructed VIX using call options and put options for period: (a) Jan., 2007 to Feb., 2009; and (b)Oct., 2008 and Dec., 2008 


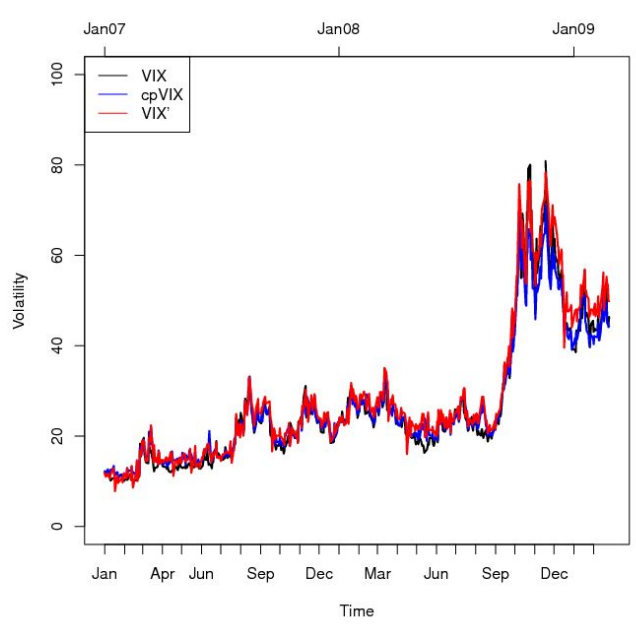

(a) Plot of the actual indices

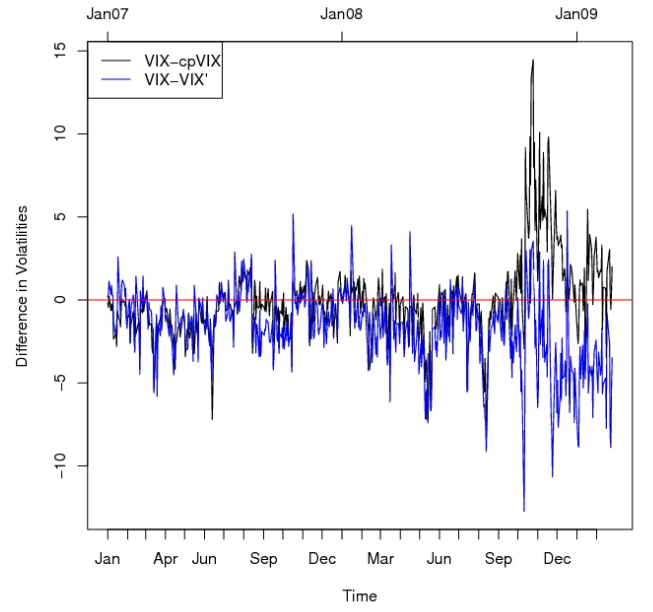

(b) Differences. End of 2009 shows higher VIX' than VIX values

Figure 3: Comparison between VIX, cpVIX and VIX'

In Table 1 we summarize all the indices constructed.

Table 1: The names of constructed VIX and data used.

\begin{tabular}{|c|l|}
\hline Type of constructed VIX & Data used \\
\hline cVIX-1 & Near term call options \\
\hline cVIX-2 & Next term call options \\
\hline cVIX-b & Both near and next term call options \\
\hline pVIX-b & Both near and next term put options \\
\hline cpVIX & Average of cVIX-b and pVIX-b \\
\hline VIX & Both near and next term call and put options \\
\hline
\end{tabular}

\subsection{Convergence of the method used in the construc- tion of indices}

We study the convergence of the results obtained using the quadrinomial tree method when computing various indices. We use different number of steps 


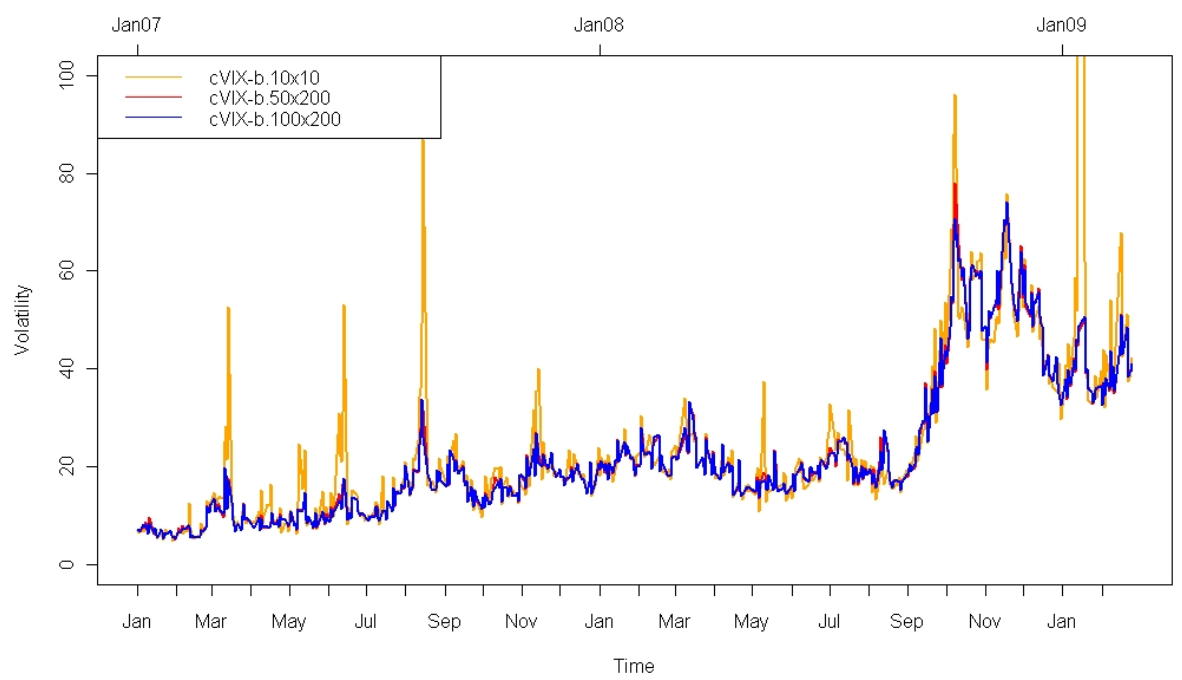

Figure 4: Convergence of constructed volatility index using quadrinomial tree method

in the tree $(10,50$, and 100 steps) as well as different number of trees $(10$, 50 , and 200 times). This is exemplified using the cVIX-b calculation. To avoid image clutter we only show a reduced number of results in Figure 4. The index converges as the trees becomes larger. This is in agreement with the theoretical results from Florescu and Viens (2008).

\subsection{Comparison between constructed VIX using call and put options chains}

In the calculation of VIX, CBOE uses the out-of-money call and out-ofmoney put options. As detailed in the description we may use any type of options and produce various types of indices. In this section, we analyze the resulting indices separately using call options and put options. Figure 2(a), presents the cVIX-b (using calls) and pVIX-b using puts in comparison to the VIX. Generally, the cVIX-b has lower values than the VIX while the pVIX-b has higher values than the CBOE index. This is perhaps best explained by Bollen and Whaley (2004) who remark that the index option market such as S\&P500 became dominated by portfolio insurers or hedgers, who routinely buy out-of-the-money and at-the-money SPX put options for 
insurance purposes. This drives up the price of put options and therefore the implied volatilities from put options tends to be higher. This leads to the differences between the constructed VIX using call options and put options.

In Figure 2(b) we zoom into turbulent financial time between Oct. and Dec. 2008. It is clear that during this period, the VIX is generally the higher than either cVIX or pVIX. This seem to indicate us that during this period the demand for put options seems to be extraordinary. We should also note that during this period of time, the market was very volatile and the observed VIX index went as high as 80. Furthermore, when looking at the difference between the call based index (cVIX-b) and the put based index (pVIX-b) the spread between these volatilities was the smallest for this particular period. In Figure 5 we showcase this feature and we observe that the spread becomes negative before the market crash and stays negative for an extended period during the crash.

\subsection{A comparison of the correlation between the con- structed indices/S\&P500 and VIX/S\&P500}

It is well documented that a negative relationship exists between the index S\&P500 and the CBOE volatility index VIX; when the S\&P500 index goes up/down, VIX tends to go down/up. In Table 2 , we present the $R^{2}$ of the linear relationship between S\&P500 and various indices. The data does not show major differences between these correlations.

Table 2: The correlation between constructed VIX or VIX to S\&P500.

\begin{tabular}{|c|c|c|c|c|c|c|c|}
\hline sp500 & VIX & cVIX-2 & cVIX-b & pVIX-2 & pVIX-b & cpVIX-b & cpVIX-2 \\
\hline$R^{2}$ & 0.7818 & 0.7944 & 0.7805 & 0.7875 & 0.7912 & 0.8004 & 0.8030 \\
\hline
\end{tabular}

\subsection{An analysis of the predictive power of the different indices}

In this section we analyze the relationship between the volatility and the return of the S\&P500. Specifically, we are interested in determining whether a significant increase in the volatility is followed by a significant drop in the 

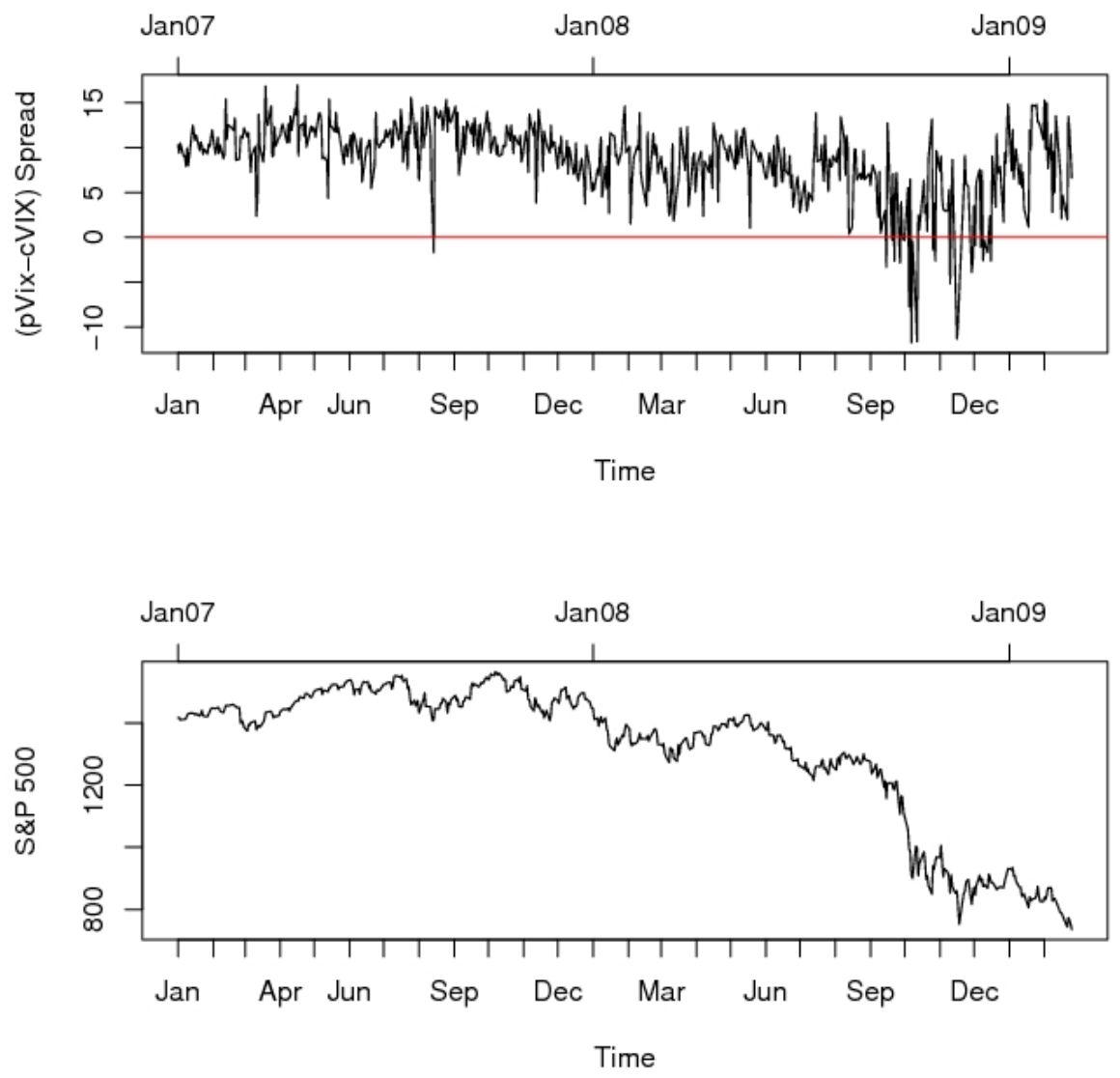

Figure 5: The difference (spread) pVIX-cVIX. A decrease in spread seems to indicate a decrease in the market index 

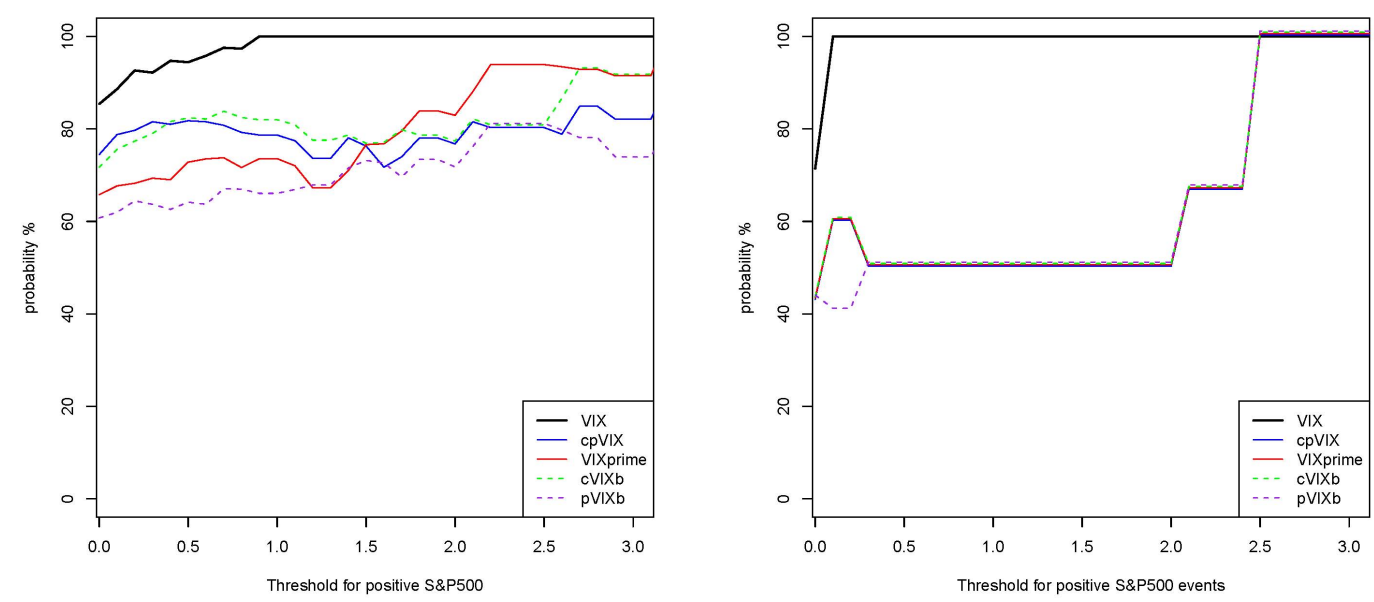

Figure 6: Probability of a positive return on S\&P500 when $d=0$

S\&P500 prices and to determine which volatility index variant prove to be a better predictor of this relationship. We analyze the same day observations as well as one day forecast. Furthermore, we investigate the relationship for a selection of observations centered around major financial events as presented in Table 3. We consider the VIX from CBOE and the following index variants: cpVIX, VIX', cVIXb, and pVIXb.

For each of these variants we calculate the rate of change and we estimate the conditional probability that S\&P500 is moving in the opposite direction.

$$
\begin{aligned}
& \operatorname{Prob}\left(\operatorname{Return}_{\mathrm{S} \& \mathrm{P} 500}<0 \mid\left(\text { Return }_{\mathrm{Vol} \text { Index }}>L, \operatorname{lag}=d\right)\right) \text {, and } \\
& \operatorname{Prob}_{\left(\text {Return }_{\mathrm{S} \& \mathrm{P} 500}>0 \mid\left(\text { Return }_{\mathrm{Vol} \text { Index }}<L, \text { lag }=d\right)\right)}
\end{aligned}
$$

where Return S\&P500 $_{\text {is the return of S\&P500, Return }}$ Vol Index is the return of the particular volatility index, $L$ is a threshold for the index return, and $d$ represents the same day for $d=0$, and the previous day for $d=1$.

The graphs presented are grouped in two sections corresponding to the same day $d=0$ or the forecast for the next day $d=1$. On all of these graphs the $x$-axis plots the respective threshold that conditions the probabilities in (6) while the $y$ axis plots the percent of days where the S\&P500 moved in the predicted direction. The left image in a figure presents all the dataset, 

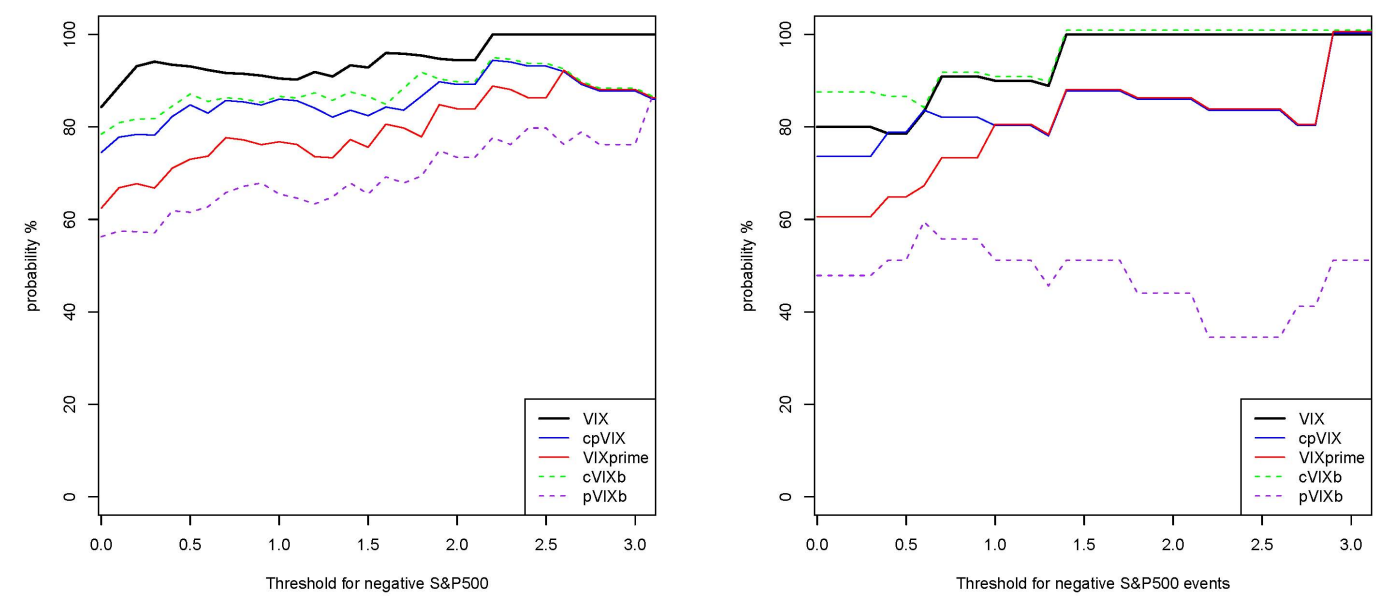

Figure 7: Probability of a negative return on S\&P500 when $d=0$

while the right image is restricted to the important financial events detailed in Table 3 on page 16 . 
Table 3: Important events that happened during Jan 07 to Feb 09 period.

\begin{tabular}{|l|l|}
\hline Central banks increase money supply & Aug. 10, 2007 \\
Countywide job slashes & Sep. 7, 2007 \\
Bush calls for economic stimulus package & Jan. 18, 2008 \\
Central European banks plan emergency cash infusion & Mar. 11, 2008 \\
Bear Stearns gets emergency funds & Mar.14, 2008 \\
J.P.Morgan to acquire Bear Stearns & Mar. 16, 2008 \\
Federal Reserve cuts rates by 0.75 & Mar. 18, 2008 \\
IMF may sell 400 tons of gold & Apr. 8, 2008 \\
Citigroup anticipates a giant loss, Fed cuts rate again & Apr. 30, 2008 \\
US backs lending firms & Jul. 13, 2008 \\
US inflation at 26 year high & Jul. 16, 2008 \\
Mortgage firms bail out & Sep. 7, 2008 \\
Lehman Brothers files for bankruptcy & Sep. 14, 2008 \\
Bush hails the financial rescue plan & Sep. 19, 2008 \\
\$700 billion package failed & Sep. 29, 2008 \\
House backs the bail out plan & Oct. 3, 2008 \\
plans to buy 125 billion stakes in banks & Oct. 14-29, 2008 \\
Citigroup cut 75000 jobs & Nov. 17, 2008 \\
Citigroup gets US Treasury lifeline & Nov. 23, 2008 \\
800 billion stimulus package announced & Nov.25, 2008 \\
Bank of America cuts 30000 jobs & Dec. 11, 2008 \\
Madoff 50 billion scandal & Dec. 13, 2008 \\
Auto industry bail-out & Dec. 19, 2009 \\
US financial sector stocks decline sharply & Jan. 20, 2009 \\
New bank bail-out & Feb. 10, 2009 \\
\hline
\end{tabular}

We analyze separately the positive and negative movement in figures 6 and 7. It is pretty clear from these images that the CBOE VIX is the best indicator for the return/volatility evolution within the same day $(d=0)$. The profile of the probability curves for the major events selection follows a similar profile to the probability curves for all data analyzed. However, the fact that an increase in the volatility index calculated from the calls indicates a drop in the S\&P500 index was a surprise to us.

For prediction purposes, we analyze the relationship between the previous 

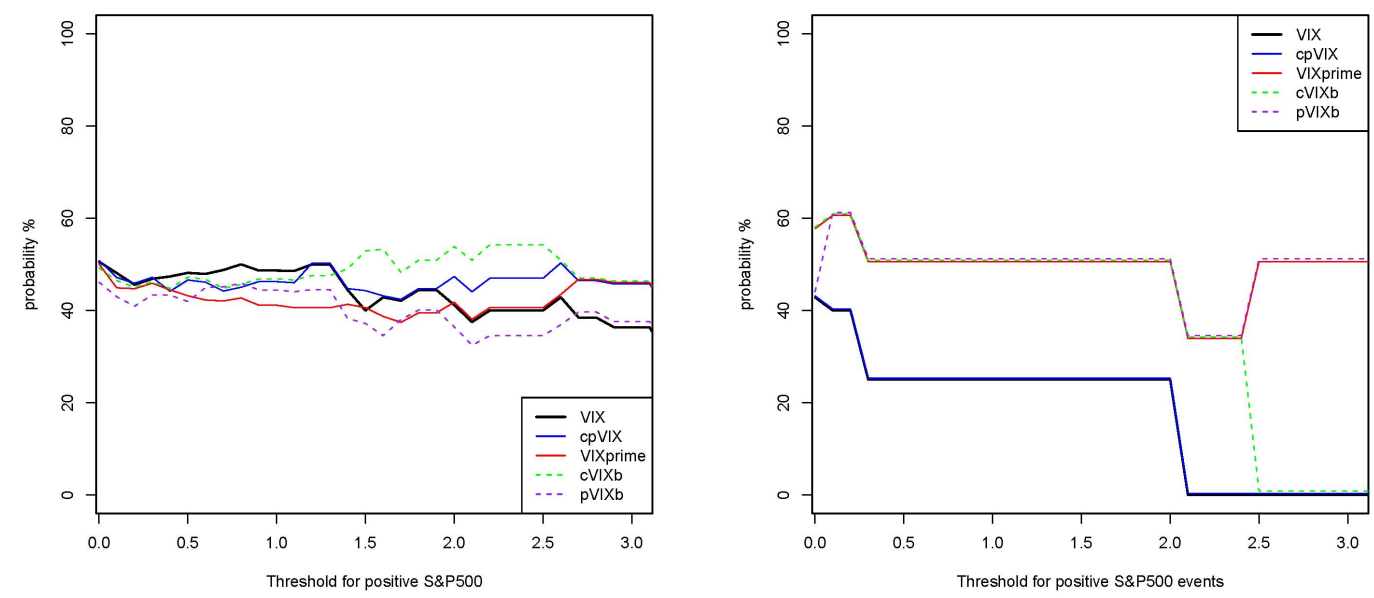

Figure 8: Probability of a positive return on S\&P500 when $d=1$

day volatility index and the return on the S\&P500 the following day. The corresponding graphs once again split by the positive and negative thresholds are presented in figures 8 respectively 9 .

This time we observe that the CBOE VIX is one of the worst indicators for future day evolution of the S\&P500. In some cases the probability of predicting a positive return correctly is well below $50 \%$.

In contrast two of the indices we calculated stand out. We note that a drop in the cVIXb (calculated from call options) forecasts with the highest probability a positive return of the S\&P500. Furthermore, an increase in the pVIXb (calculated from the put options) has a clear advantage in forecasting second day negative S\&P500 returns. The probabilities for the pVIXb are in fact very high for all data sample and also for the major events in the market considered in this analysis.

We hope that we convinced the reader that using different types of options in the volatility index calculation has the potential to reveal more information about the market than the VIX. 

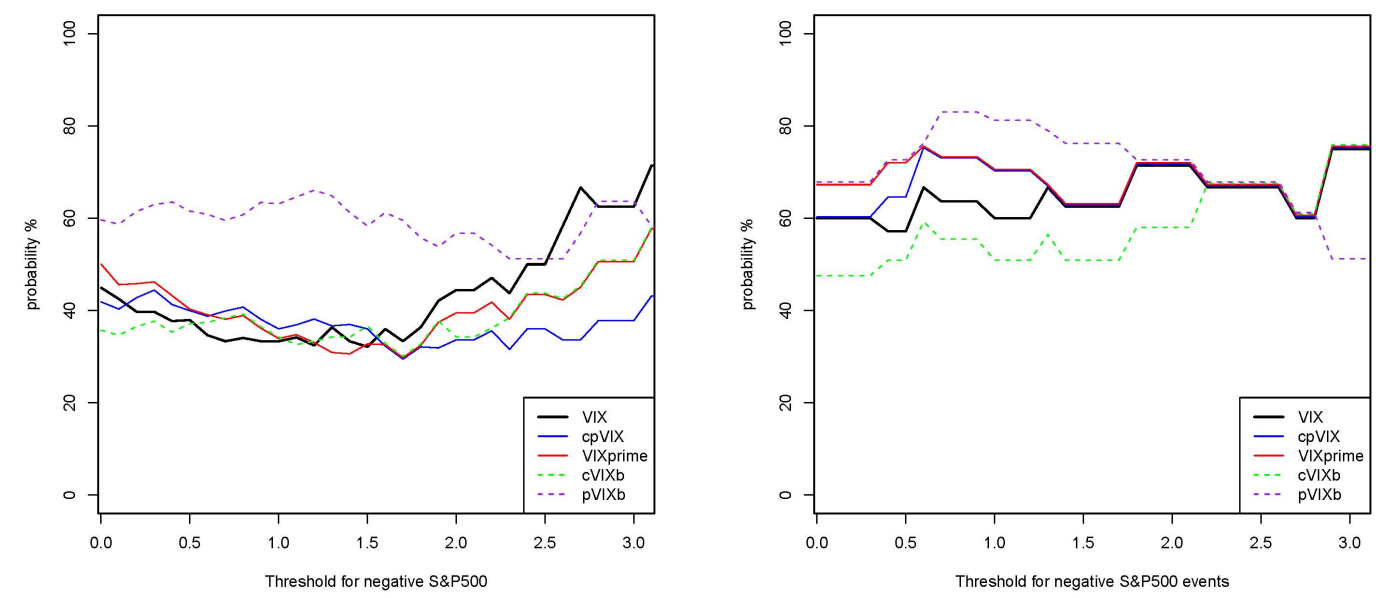

Figure 9: Probability of a negative return on S\&P500 when $d=1$

\section{Summary and Conclusion}

We propose a new methodology of calculating a value that represents the market volatility at a given moment in time by implementing a stochastic volatility technique. We believe this technique is a viable way to produce a market index. We propose several variants of such indices and we believe each of them is valuable as an indicator of a market movement. The index constructed from calls (cVIX-b) may be considered as an indicator of market's positive movement while the index constructed from put options (pVIX-b) as an indicator of future negative movement in the market. The difference (spread) between the two indices may be indicative of future market movement. The average of these two indices (cpVIX) and the index constructed with all options (VIX') both have value in determining when the VIX undervalues or overvalues the market volatility.

Finally, we analyze the relations between all these types of indices. We believe all of them bring more information about the market and the methodology has the potential to produce market indicators each indicative of a certain aspect of the financial market. 


\section{Appendix A: Step-by-Step Explanation of the CBOE Procedure for Calculating VIX Index}

Using data obtained at the market close on Sept 8, 2009, we replicate the VIX value following the CBOE procedure. First, we need to do some calculation and re-arrangement of the data. The procedure is described below.

1. Selection of options chains. VIX generally uses put and call options in the two nearest-term expiration months while there are more options chains trading in the market. Also, it should be pointed out that, with 8 days left to expiration, the VIX "rolls" to the second and third contract months in order to minimize the pricing anomalies that might occur close to the maturity.

2. $T$, the time to expiration, is measured in minutes rather than in days. Specifically, the calculation of $T$ is given by the following expression:

$$
T=\left\{M_{\text {currentday }}+M_{\text {settlementday }}+M_{\text {otherdays }}\right\} / M_{\text {year }}
$$

Where:

$M_{\text {currentday }}$ is the number of minutes remaining until midnight of the current day, $M_{\text {settlementday }}$ is the number of minutes from midnight until 8:30 a.m. on SPX settlement day, and $M_{\text {otherdays }}$ is the number of minutes in all the days between current day and the settlement day, and $M_{\text {year }}$ is the number of minutes in a year.

3. Calculating the at-the-money strike. This is done by finding the strike price at which the difference between the call and put prices is the smallest.

4. Calculation of $F$, the forward index level. This is based on the previously determined at-the-money option prices and the corresponding strike price:

$$
F=\text { StrikePrice }+e^{r T}(\text { CallPrice }- \text { PutPrice })
$$

Note that since two options chains are used in the calculation, two

forward index level should be obtained, for the near term and next term options chains. 
5. Selection of $K_{0}$, the strike price immediately below the forward index level, $F$.

In the following, we demonstrate how to obtain the VIX value using CBOE procedure using data obtained when the S\&500 options stopped trading at 3:15PM (Middle time) on Sept 8, 2009.

Step 1: Calculate the time to expiration $T$, forward index level $F, K_{0}$, the strike price immediately below $F$, and data arrangement:

$T_{1}=\left\{M_{\text {currentday }}+M_{\text {settlementday }}+M_{\text {otherdays }}\right\} / M_{\text {year }}$

$=(525+510+12960) / 525600$

$=0.026626712$

$T_{2}=\left\{M_{\text {currentday }}+M_{\text {settlementday }}+M_{\text {otherdays }}\right\} / M_{\text {year }}$

$=(525+510+53280) / 525600$

$=0.103339041$

We should note that the total days in a year is 365 days. Since the smallest difference between call and put price is at strike $\$ 1025$, the at-the-money strike is determined to be at $\$ 1025$ for both near term options and next term options. Therefore, using federal funds effective rate at $0.15 \%$, the forward index level $F_{1}$ for the near term options and forward index level $F_{2}$ for the next term options are:

$F_{1}=$ StrikePrice $+e^{r T_{1}}$ (CallPrice - PutPrice)

$=1025+e^{0.0015 \times 0.026626712}(13.25-13.9)=1024.35$

$F_{2}=$ StrikePrice $+e^{r T_{2}}$ (CallPrice - PutPrice $)$

$=1025+e^{0.0015 \times 0.103339041}(29.15-30.35)=1023.80$

We also obtain $K_{0}$, the strike price immediately below $F$, which is $\$ 1020$ for both expirations. Then we select call and put options that have strike prices greater and smaller, respectively, than $K_{0}$ (it is 1020 here) and non-zero bid price. After encountering two consecutive options with a bid price of zero, do not select any other options. Note that the prices of the options are calculated using the midpoint of the bid-ask spread. At $K_{0}$, the average of call and put price is used. The data selected is summarized below:

- Calculation of time to maturity:

$T_{1}=0.026626712$

$T_{2}=0.103339041$

- At-the-money strike: $\$ 1025$ for both near term options and next term options. 
- Federal funds effective rate: $0.15 \%$.

- The forward index level $F_{1}$ for the near term options and forward index level $F_{2}$ for the next term options are:

$F_{1}=1024.35$

$F_{2}=1023.80$

- $K_{0}$, the strike price immediately below $F$, is $\$ 1020$ for both expirations.

Step 2: Calculate the volatility for near term and next term options. We apply the following equations to calculate the VIX to the near term and next term options:

$$
\begin{aligned}
& \sigma_{1}^{2}=\frac{2}{T_{1}} \sum_{i} \frac{\Delta K_{i}}{K_{i}^{2}} e^{r T_{1}} Q\left(T_{1}, K_{i}\right)-\frac{1}{T_{1}}\left(\frac{F_{1}}{K_{0}}-1\right)^{2} \\
& \sigma_{2}^{2}=\frac{2}{T_{2}} \sum_{i} \frac{\Delta K_{i}}{K_{i}^{2}} e^{r T_{2}} Q\left(T_{2}, K_{i}\right)-\frac{1}{T_{2}}\left(\frac{F_{2}}{K_{0}}-1\right)^{2}
\end{aligned}
$$

We need to pay attention to value of $\Delta K_{i}$. Generally $\Delta K_{i}$ is half the distance between the strike on either side of $K_{i}$, but at the upper and lower ends of any options chain, $\Delta K_{i}$ is simply the distance between $K_{i}$ and the adjacent strike price. We obtain: $\sigma_{1}^{2}=0.055576664$ and $\sigma_{2}^{2}=0.066630428$.

Step 3: Interpolate $\sigma_{1}^{2}$ and $\sigma_{2}^{2}$ to get a single value with a constant maturity of 30 days. VIX is 100 times of the square root of this value.

$$
\sigma^{2}=\left\{T_{1} \sigma_{1}^{2} \frac{N_{T_{2}}-N_{30}}{N_{T_{2}}-N_{T_{1}}}+T_{2} \sigma_{2}^{2} \frac{N_{30}-N_{T_{1}}}{N_{T_{2}}-N_{T_{1}}}\right\} \frac{N_{365}}{N_{30}}
$$

where

$N_{T_{1}}$ is the maturity of the near term options in minutes (13995), $N_{T_{2}}$ is the maturity of the next term options in minutes (54315), $N_{30}$ is the maturity of a 30-day options in minutes $(43,200)$, $N_{365}$ is the number of minutes in a year $(525,600)$.

Therefore, the $V I X=100 \times \sigma=25.62$, which is exactly the same value as the one provided by $\mathrm{CBOE}$. 


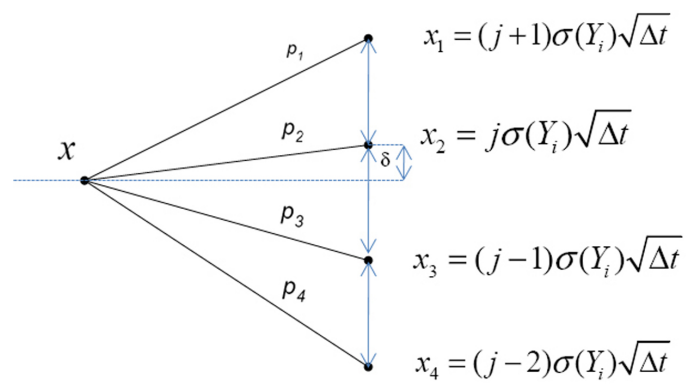

Figure 10: Schematic of one step in the quadrinomial tree method.

\section{Appendix B: Explanation of the new volatility index calculation}

The following gives details about the quadrinomial tree approximation and the new volatility estimation. In Figure 10 we present a one step construction. Assume that we are given an (empirical or theoretical) distribution for the stochastic volatility process at the time $t$ when pricing is done. Sample from this volatility distribution to obtain the value $\varphi$. Given this value we construct a grid of points of the form $l \varphi \sqrt{\Delta t}$ with $l$ taking integer values. No matter where the parent $x$ is, it will fall at one such point or between two grid points. In this grid, let $j$ be the integer that corresponds to the point right above $x$. Mathematically, $j$ is the integer equal to the integer part of $\frac{x}{\varphi \sqrt{\Delta t}}+1$, as $x_{3}<x<x_{2}$. We will have two possible cases: either the point $j \varphi \sqrt{\Delta t}$ on the grid corresponding to $j$ (above) is closer to $x$, or the point $(j-1) \varphi \sqrt{\Delta t}$ corresponding to $j-1$ (bellow) is closer. We use $\delta$ to denote the distance from the parent $x$ and the closest successor on the grid. We use $q$ to denote the standardized value, i.e.

$$
q:=\frac{\delta}{\varphi \sqrt{\Delta t}}
$$

There are two cases: first when $x_{2}$ is closer to $x$ and the second when $x_{3}$ is closer to $x$. In the first case by considering the mean of the increment converging to the drift of the process $X_{t}$, the probabilities corresponding to 
each of the points on the grid can be calculated as:

$$
\begin{aligned}
& p_{1}=\frac{1}{2}\left(1+q+q^{2}\right)-p \\
& p_{2}=3 p-q^{2} \\
& p_{3}=\frac{1}{2}\left(1-q+q^{2}\right)-3 p \\
& p_{4}=p
\end{aligned}
$$

In the second case, when $x_{3}$ is closer to $x$ the probabilities are:

$$
\begin{aligned}
& p_{1}=p \\
& p_{2}=\frac{1}{2}\left(1+q+q^{2}\right)-3 p \\
& p_{3}=3 p-q^{2} \\
& p_{4}=\frac{1}{2}\left(1-q+q^{2}\right)-p
\end{aligned}
$$

where $p \in\left[\frac{1}{12}, \frac{1}{6}\right]$. It is observed that when $p$ is close to $\frac{1}{6}$ the option values obtained are stable even with few replications (Figure 4 in Florescu and Viens (2008)). In this paper we set $p=0.135$ throughout the algorithm.

\section{Step-by-Step Explanation of the Construction of VIX Using Stochastic Volatility Quadrinomial Tree Method}

Here we use quadrinomial tree model to compute the price of a synthetic options with exact 30 days maturity using distribution of implied volatility obtained from S\&P500 as input. Then by Black and Scholes (1973) formula, we obtain the implied volatility of this synthetic option. We want to study whether or not this implied volatility multiplied with 100 can better reflect the market volatility.

There are four steps in the construction of this volatility index:

- Compute the implied volatilities of entire option chain on SP500 and construct an estimate for the distribution of current market volatility. The implied volatility is calculated by applying Black-Scholes formula.

- Use this estimated distribution as input to the quadrinomial tree method. Obtain the price of an at-the-money synthetic option with exactly 30 day maturity.

- Compute the implied volatility of the synthetic option based on BlackScholes formula once the 30-day synthetic option is priced. 
- Obtain the estimated volatility index by multiplying the implied volatility of the synthetic option by 100 .

Please note that the most important step in the estimation is the choice of proxy for the current stochastic volatility distribution.

\section{References}

Black, F. and M. Scholes (1973). The valuation of options and corporate liability. Journal of Political Economy 81, 637-654.

Bollen, N. and R. Whaley (2004, Apr.). Does net buying pressure affect the shape of implied volatility functions? Journal of Finance 59(2), 711-753.

CBOE (2003). The new cboe volatility index-vix. white papers, CBOE.

Demeterfi, K., E. Derman, M. Kamal, and J. Zou (1999, March). More than you ever wanted to know about volatility swaps. Technical report, Goldman Sachs Quantitative Strategies Research Notes.

Florescu, I. and F. Viens (2008). Stochastic volatility: option pricing using a multinomial recombining tree. Applied Mathematical Finance Journal 15, $151-181$.

Jiang, G. J. and Y. S. Tian (2007). Extracting model-free volatility from option prices: An examination of the VIX index. Journal of Derivatives $14(3), 35-60$. 